\title{
Gradhiva
}

GRADHIV

Revue d'anthropologie et d'histoire des arts

18 | 2013

Le monde selon l'Unesco

Faulkner. Le nom, le sol et le sang

Gaetano Ciarcia

(2) OpenEdition

Journals

Édition électronique

URL : http://journals.openedition.org/gradhiva/2756

DOI : 10.4000/gradhiva.2756

ISSN : 1760-849X

Éditeur

Musée du quai Branly Jacques Chirac

Édition imprimée

Date de publication : 1 décembre 2013

Pagination : 234-241

ISBN : $978-2-35744-072-2$

ISSN : 0764-8928

Référence électronique

Gaetano Ciarcia, «Faulkner. Le nom, le sol et le sang », Gradhiva [En ligne], 18| 2013, mis en ligne le 01 décembre 2016, consulté le 22 septembre 2020. URL : http://journals.openedition.org/gradhiva/2756 ; DOI : https://doi.org/10.4000/gradhiva.2756

(c) musée du quai Branly 


\section{Faulkner. Le nom, le sol et le sang}

par Gaetano Ciarcia

Jean Jamin, Faulkner. Le nom, le sol et le sang.

Paris, CNRS Éditions, 2011.

Dans la réflexion de Jean Jamin, l'œuvre de William Faulkner occupe une place initiatique. L'auteur de Faulkner. Le nom, le sol et le sang précise en effet que le roman Lumière d'août a été, avec L'Afrique fantôme de Michel Leiris, l'une des lectures qui ont déterminé son choix d'être ethnologue. Ce moment inaugural fait ici l'objet d'une mise en perspective où les résultats produits par une fréquentation de longue durée d'un corpus littéraire croisent et prolongent une entreprise de conceptualisation de notions anthropologiques ${ }^{\mathbf{1}}$.

Faulkner (1897-1962) entretint avec le milieu au sein duquel il vécut et sur lequel il écrivit, celui d'une société du sud des États-Unis, une relation marquée par une sorte d'excès de proximité. Ce monde, fait de la séparation brutale et intime entre Blancs et Noirs, se confond avec sa production narrative qui, en le récréant, s'en inspire et s'en détache d'une manière implicitement et anthropologiquement critique. Ici, tout sentiment de domesticité des origines s'estompe au profit du regard trouble que l'auteur porte sur la force des liens sociaux et familiaux que Jamin a choisi d'interroger, dont ceux définis par les thèmes du sang, du sol et du nom.

Décomposée durant la guerre civile qui l'avait opposée aux États abolitionnistes du Nord, la société blanche sudiste à laquelle appartient Faulkner est minée, note Jamin, par le viol originel dont elle est issue, à savoir la spoliation par les colons des terres des Indiens. Cette société désormais vaincue est habitée par les illusions de ceux qui se réclament explicitement ou tacitement d'une noble ascendance alors que le passé de la conquête territoriale et de l'économie esclavagiste (ainsi que le présent de la ségrégation dont les Noirs sont les victimes) l'empêche de croire vraiment en l'âge d'or d'une autochtonie heureuse héritée. Néanmoins, les «Blancs» qui agissent (parfois en attendant) dans les récits faulknériens se veulent appartenir à une «race» prétendument pure face au péril obsédant de sa profanation par sa partie maudite et vis-à-vis du mutisme maudissant d'une humanité "esclave», contigüe et séparée ${ }^{2}$. Ils sont aux prises avec l'appréhension vécue et racontée d'une condition à la fois paradoxale et banale, propre à toute société post-esclavagiste, où l'altérité dominée participe fondamentalement de l'image que la «caste» dominante a ou veut avoir d'elle-même. Ici, les représentations mentales du Noir et de la couleur noire sont nécessaires aux damnés d'une logique lignagère qui s'efforce et peine à se perpétuer. Car si, comme le dit Jamin, dans le monde de Faulkner pour les Blancs tout est déjà là, les Noirs eux, endurent et durent. Privés de passé, ces derniers ne peuvent que se projeter par inertie douloureuse dans un avenir qui, en revanche, est inatteignable pour les rejetons blancs enchaînés à leur généalogie fantôme.
1. Pour quelqu'un, comme le rédacteur de ces notes, dont le parcours de recherche a été lui auss inspiré par le journal de voyage de Michel Leiris ainsi que par les travaux de Jean Jamin, écrire un compte rendu du texte de ce dernier sur William Faulkner relève d'une sorte de modeste et personnel défi «généalogique».

2. $\mathrm{Si}$, dans de nombreuses sociétés observées par les ethnologues, le silence peut être l'expression de "paroles " muettes qui transmettent des messages de domination, le lecteur des travaux de Jamin pourra retrouver, à l'échelle de la société ségrégationniste du sud des États-Unis, un questionnement déclinant une autre forme des lois $d u$ silence que, en contrepoint de la présence assujettie des Noirs, la prolifération de la parole blanche écrite mise en récit par Faulkner raconte et recèle. Voir Jean Jamin, Les Lois du silence. Essai sur la fonction sociale du secret Paris, Maspero, 1977. 


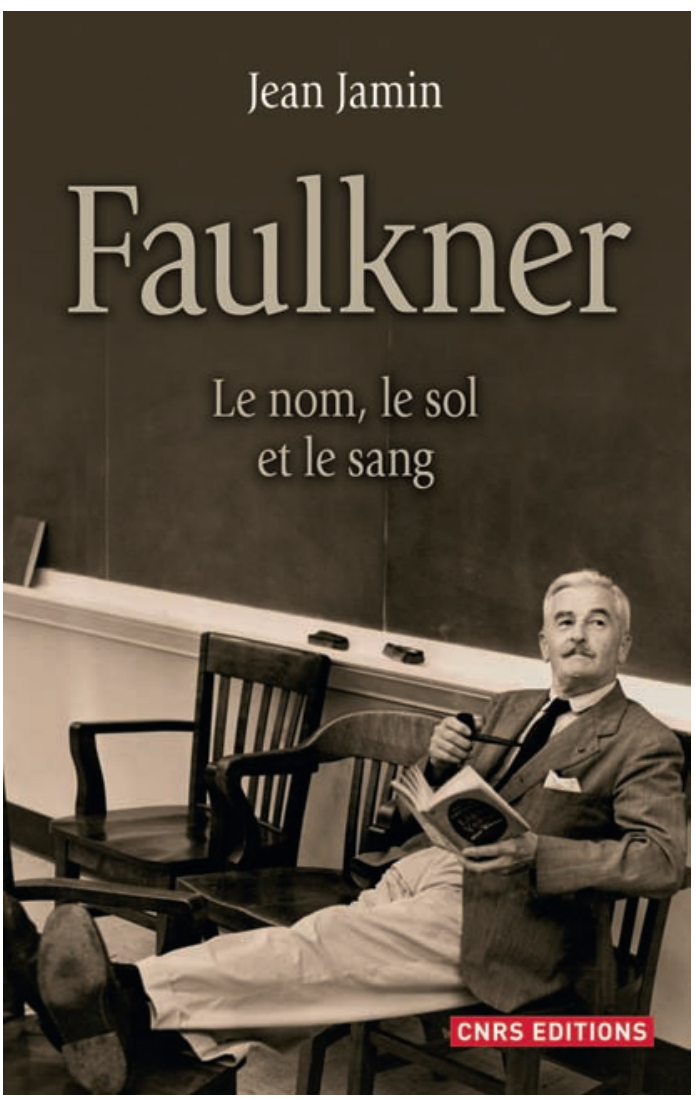

Le livre de Jamin nous suggère que, dans l'enchevêtrement biobibliographique entre récits "vécus" et narration «jouée», la condition d'éloignement provoquée par l'acte de l'écriture cohabite d'une manière tumultueuse avec la restitution dramatique de formes de vie propres au groupe humain que le romancier connaît affectivement de l'intérieur. Les choses que Faulkner raconte, tout en étant le résultat de sa création, sont déjà advenues quelque part dans son expérience imaginative et vernaculaire; il est même possible de dire que, comme pour tous les habitants de son pays en papier, elles l'ont précédée. Car, au contraire de Marcel Proust, chez Faulkner le passé n'est pas une terre devenue étrangère qu'il s'agirait de «retrouver", mais plutôt une donnée immanente aux vies qu'il invente et raconte. Une telle antériorité, prenant parfois le sens de destinée, imprègne les formes de la présence au monde et à soi de ses personnages qui semblent, dès ou par leur naissance, être irrémédiablement pris dans les filets d'une histoire fatale. Nous accédons ainsi à la réalité anthropologique des faits fictionnels d'une région littéraire, celle du comté de Yoknapatawpha, calqués sur les «mœurs» et le comté bien réels de Lafayette, Mississippi.

Les sagas faulknériennes peuvent être lues comme des variations et des réécritures, sur les modes de la vraisemblance et de la métamorphose, d'un univers social que l'écrivain a vu de son vivant depuis sa double condition d'«indigène" et d'observateur. L'anthropologue semble alors voir dans la fiction un lieu de dramatisation du quotidien ou de l'ordinaire, un outil permettant de problématiser et d'appréhender la stabilité apparente des sociétés qu'il étudie. Jamin montre ainsi comment l'œuvre qu'il interroge avec finesse et originalité génère des significations et des jugements sur une réalité locale, inventée, que le regard de l'observateur-créateur qu'est Faulkner reflète tout en la métamorphosant.

Dans l'avant-propos, Jamin s'interroge sur le statut de la fiction et remarque qu'«à la différence de la connaissance objective qui est partielle, provisoire, approchée, réfutable, la fiction n'accepte ni demiteinte, ni demi-mesure: elle est réussie ou ratée, mais elle ne se fonde pas sur des critères permettant de distinguer le vrai du faux» (p. 14). II attribue ainsi à l'illusion littéraire la qualité de «"donner à voir" autrement» (p. 21) susceptible de conférer - pourrions-nous en conclure - une strate herméneutique complémentaire à toute tentative objectivante de description. Chez Faulkner, le donner à voir autrement se ferait par des détours, quasi concentriques, à travers l'étendue géographiquement limitée du territoire du comté de Yoknapatawpha et la profondeur archéologique des intrigues - insondables pour ses propres acteurs -, des lignées et des destins dont peuvent témoigner quelques-unes des généalogies conçues par l'écrivain américain, reconstituées et reproduites en annexe de Faulkner. Le nom, le sol et le sang. Jamin signale d'ailleurs qu'à l'échelle générale de l'œuvre faulknérienne, "le but n'est pas ici de simuler le réel mais de mettre au jour ses rouages» (p. 22). Un tel "effet de réel» produit un effet d'exotisme qui me semble aller de pair avec une heuristique «sauvage» de ce qui est trop connu. II ne s'agit pas seulement de réfléchir à ce que l'écriture littéraire fait des/aux pratiques et apparences de la stabilité sociétale, mais de penser comment la fiction bricole une sorte d'intensification du particulier et de 
ses méandres en l'altérant, en le mettant sous observation, donnant lieu à une distanciation créatrice et devenant le lieu des possibles. Reprenant une remarque de Maurice Merleau-Ponty dans Éloge de la philosophie, Jamin parle ici d'une "variation imaginaire" des rapports sociaux qui, à l'aune de la fiction, "recevront une signification neuve ou feront apparaître un ordre caché, offrant dès lors mieux que des idées: des "matrices d'idées" [...]" (p. 23). Par ailleurs, il souligne que c'est l'univers romanesque qui est exotique et non pas les lieux ayant inspiré la narration: "Étrange altérité, ai-je dit, car c'est bien avec du même que, chez Faulkner, se fabrique de l'autre» (p. 28). Cette «étrange altérité», qui est aussi le titre du premier chapitre, suggère au lecteur que la fiction fait plus que donner à voir autrement, elle donne aussi à penser autrement. Une telle possibilité implique le lecteur dans une relation entre la fiction de soi-même et l'avènement, dans sa pensée, de l'autre-soi car, en lisant Faulkner, il peut se dire: «[...] je suis ce que je lis du devenir de l'autre; la réciproque étant également possible: je suis ce que l'autre lit de mon devenir [...]” (p. 33). Car, si «l'identité et l'altérité ne sont, ne seront jamais stables: pas plus le nom que le sol ou le sang ne servent à les fixer durablement, non plus que le "papier" qui les a couchées et mises en récit [...]” (p. 35), leur relation vécue et irrésolue, voire leur dialectique, est au cœur de la société que Faulkner explore et dans laquelle il est pris: son incapacité à s'affranchir hors littérature, dans la vie civile, des préjugés de la race en est la preuve. En effet, alors que ses fictions inoculent constamment le doute sur les aberrations et les idioties de l'identité, son vécu l'empêche de s'extraire de la gangue raciste affectant les lieux où il est né et qu'il raconte comme s'il s'agissait d'un ailleurs. Logique anti-ethnocentrique et anti-égocentrée du détour nécessaire aussi bien au romancier qu'à l'ethnographe.

Dans le deuxième chapitre intitulé «Retour à Rowan Oak", du nom que Faulkner donna au lieu-dit de sa maison par référence à une ancienne cérémonie celte, Jamin explique que tout comme les esclaves noirs, inventeurs de negro-spirituals et de gospel songs, «Faulkner détourne et réinvente les mythes, religieux ou profanes, anciens ou nouveaux, transplantés ou créés de toute pièce pour refonder la société, y repenser la place de l'individu, recommencer l'Histoire" (p. 51). Mais tout comme les personnages blancs de ses romans, il ne parvient pas à faire recommencer l'Histoire, ayant conscience que les fondations de son monde sont, comme Jamin l'évoque, en passe de s'envoler ou déjà emportées par le vent. En ce sens son racisme, absent de son écriture romanesque mais attesté dans ses conduites et ses prises de position, est le signe d'une impossibilité à dépasser le mythe de la pureté des origines. Pourtant ses fictions «sonnent juste» (Jamin) parce qu'elles arrivent à donner à voir et à penser les effets diffus de la violence et du viol coloniaux, l'absurdité de la logique racialiste, c'est-à-dire l'impureté du commencement. De manière pertinente, Jamin montre qu'il s'agit d'une "sorte de modèle réduit d'une société obsédée par l'idée même de société, de ses récits de fondation, de ses actes de régénérescence, ou des signes de sa dégénérescence" ( $p .52)$. Cette société prend très au sérieux les modes parfois caricaturaux de son existence, ses apparences vécues comme les formes immémoriales d'une essence. Les façades de la respectabilité du maitre et de sa demeure ainsi que de la soumission de l'esclave dissimulent la cage de l'«étrange intimité qui, de fait, avait durablement 


\section{ci-contre}

fig. 1

Andrew Wyeth,

James Loper, 1952, tempera sur toile. (C) Andrew Wyeth. Collection Brandywine River Museum, Gift of Mary Carey Haskell, 1971. lié des populations blanches et noires, mais, dans un même mouvement, les avait séparées de la manière la plus insensée, la plus inique et la plus brutale, les emmurant dans leur épiderme» (p. 62). Chez Faulkner, ces relations oralement presque indicibles sont explorées par écrit dans leurs spirales et leurs abîmes auxquels Jamin restitue une densité et un relief ethnologiques à travers son attention pour les interactions dramatiques entre les notions cruciales du sang, du sol et du nom.

En ce qui concerne la question croisée du nom et du sol, l'anthropologue souligne que, bien que n'exprimant «rien d'autre que lui-même» (p. 80), le nom intervient chez Faulkner comme un marqueur identitaire. Au lieu d'asseoir la logique de l'appartenance collective, il l'interroge dramatiquement à partir de l'altérité dont le soi de chaque individu serait le vecteur («je est un autre») et dont le nom serait le masque. Ainsi, à une échelle à la fois singulière et communautaire, il se détache, pièce maîtresse de toute intrigue ou, comme le rappelle Jamin, intrigue première dont témoigne l'incipit melvillien dans Moby Dick: «Call me Ishmael.» Et tout comme il n'y aurait pas de lien originel et de correspondance primordiale entre le nom et celui qui le porte, chez Faulkner «il n'y a pas de territoire vierge, pas de paysage qu'aucun regard n'eût balayé, pas de sol qu'aucun pied n'eût foulé, ni d'étendue qu'aucune langue n'eût dénommée - pas de point d'eau qu'aucune bouche n'eût souillé. Donc, pas de nature originelle, pas de nature inviolée. Jadis, au commencement, peut-être n'y eut-il rien, mais c'est un rien que... qui implique un quelque chose » (p. 108). Et s'il n'y a pas de nom propre à chaque individu, tout comme le paysage des origines le nom est le fantôme d'une identité (de son imposition par autrui, de sa quête par soi-même), entre la présence à soi et le monde, impossible à retrouver et à rétablir. À l'instar de la question onomastique, dans la partie où Jamin aborde le rapport entre les thèmes du sang et du sol, le premier est pensé dans sa contiguïté avec le problème de la race, s'exprimant entre autres dans la fameuse règle de "la goutte de sang" (noir) qui suffit à salir la pureté de la race blanche. L'histoire familiale racontée dans Absalon, Absalon! est un exemple significatif de cette "hantise du métissage" (p. 152) dans l'œuvre faulknérienne. Du reste, le génie de l'écrivain inscrit la figure et la condition des Noirs au sein de "la conscience des Blancs" et il arrive à «mettre ceux-ci, par leur seule présence, en face du mal qui les ronge, si ce n'est de les mettre au défi de comprendre la malédiction qui pèse sur eux, et dont ils (les Noirs) sont à la fois sujets et objets » (p. 150).

La fiction fait également œuvre d'anthropologie autour des notions de sang et de nom. Après avoir remarqué "qu'en prévenant tout accident généalogique (le métissage), le repli sur soi, qui, d'une certaine manière, est en totale contradiction avec l'esprit de la Frontière, ne peut que provoquer à terme un accident généalogique, une dégénérescence, autrement dit une tragédie dans la descendance» (p. 161), Jamin établit une comparaison entre l'endogamie "dégénérative» par laquelle la société blanche sudiste des romans de Faulkner semble se perpétuer - en s'exténuant (au contraire des Noirs qui, eux, endurent et durent) - et la théorie de la parenté dite de la maison à étages élaborée, entre autres, par Claude Lévi-Strauss à partir d'exemples ethnographiques amérindiens, où la filiation est une forme sociale de l'alliance et vice versa. Dans le monde faulknérien fait d'ombres 


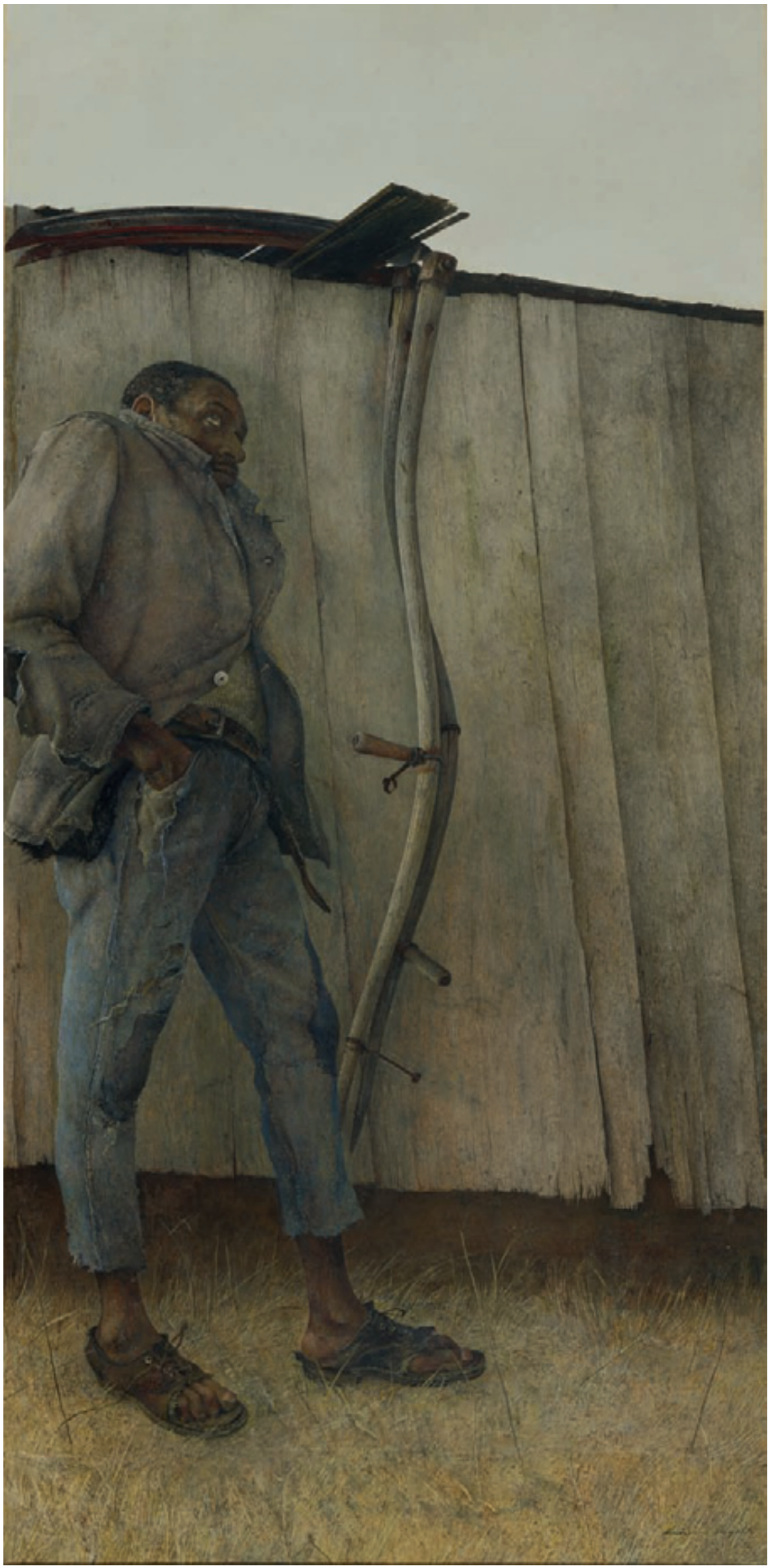




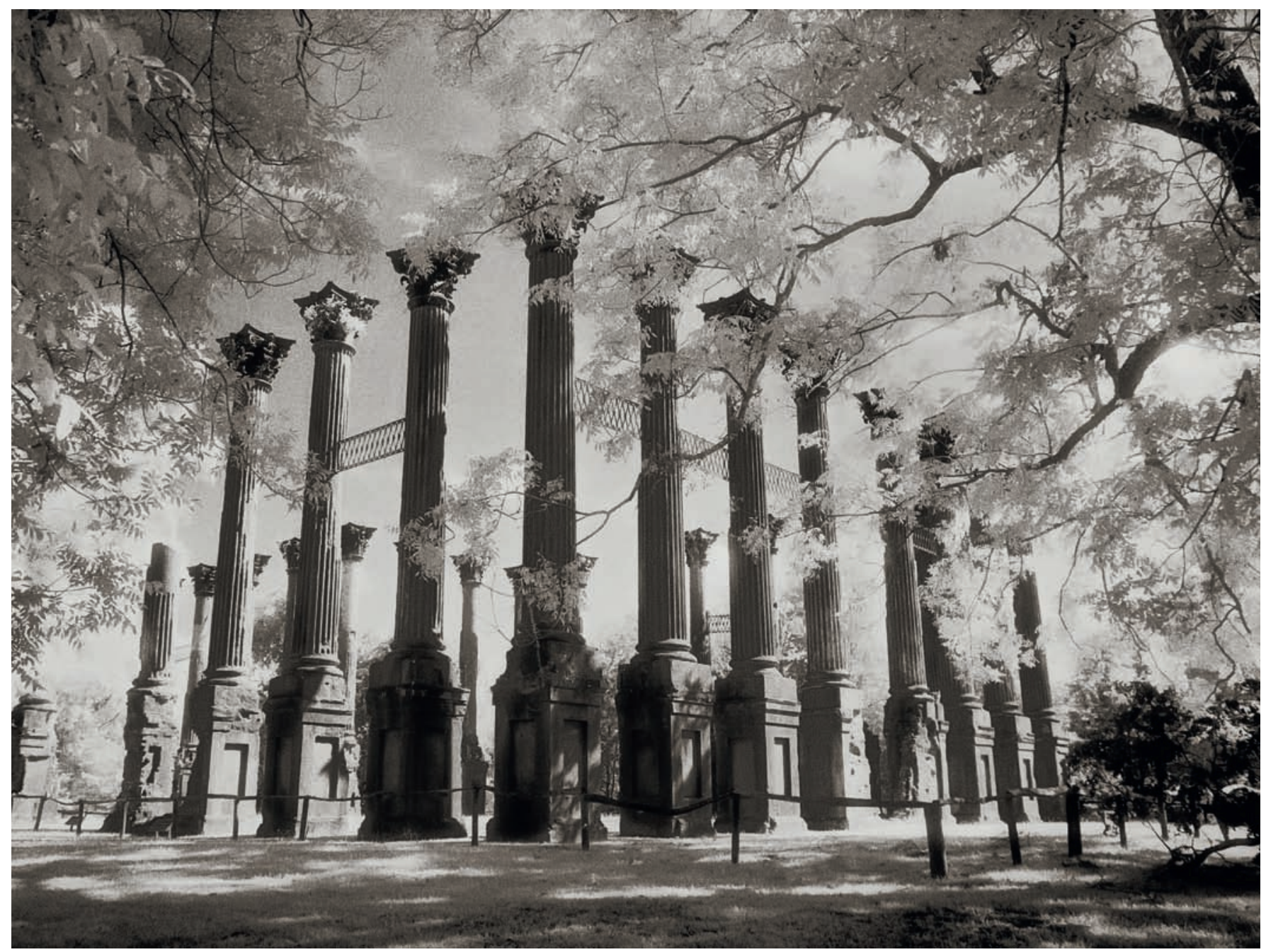

page 234 et ci dessus

fig. 2

Les ruines de Windsor

Alcorn, Mississippi, 2006.

(C) Photographie Arthur

Drooker. 
et d'apparences, la maison familiale serait le lieu où se joue le théâtre des origines et de leur prétendu devenir «naturel». Néanmoins, comme le souligne l'auteur, cette maison "est aussi un rêve - d'enracinement, de dynastie, de longévité - et, comme tout rêve, peut basculer dans le cauchemar» (p. 162). Car, malgré une présence noire vainement appréhendée comme végétale ou animale par les Blancs, l'enceinte «familiale» est faite d'irrémédiables naissances et parties communes. Dans un décor - qui devient du carton-pâte dès qu'on le scrute à la loupe de la durée historique - où les habitants miment la paradoxale noblesse européenne de leur enracinement américain, la «présence d'une population noire [...] dans le périmètre autant physique que sociologique de la "maison" " ne peut «qu'amener leurs propriétaires à faire prévaloir le "nom de la race" sur le "nom de la terre", par conséquent le droit du sang sur celui du sol » (p. 163). Sur ce sol, rappelons-le, il est socialement admis que des Noires allaitent et/ou accouchent des enfants d'hommes blancs - des enfants que, dans ce second cas, la règle de la goutte de sang transforme en Noirs -, mais il est inadmissible que des Blanches accouchent des enfants d'hommes noirs. Alors, comme «le lait et le sperme ne se résolvent pas de la même manière dans le sang » (p. 195), on pourrait se demander si cette même ligne entre Blancs et Noirs, censée empêcher à jamais l'alliance sur le même sol avec les autres qui l'occupent, ne remet pas finalement en question une règle d'échange considérée comme primaire par de nombreux anthropologues, à savoir celle de l'interdit de l'inceste. Jamin remarque que «les héros faulknériens disent pourtant quelque chose d'une vérité, de leur vérité: avoir voulu fonder une communauté unique et monochrome grâce aux barrières raciales qu'ils avaient édifiées et sans cesse renforcées, mais qui n'eut aucune autre alternative que de semer des bâtards» et ajoute: «Le droit du sang s'est donc ici superposé au droit du sol, et Faulkner traque de façon magistrale, jusque dans ses contradictions et ses aberrations, cette quasi-loi naturelle sur laquelle s'est fondée la société sudiste et peut-être au-delà toute la société américaine en dépit de son idéologie du melting-pot» (p. 194). Ici, comme nous le suggère Faulkner. Le nom, le sol et le sang, les conventions morales et mentales de la filiation, de la race et de l'autochtonie se révèlent, dans la narration romanesque, l'expression «incestueuse» de la relation interdite avec l'autre. Le sang qui devrait laver le sol est alors le terrain littéraire de la vérité anthropologique d'un exotisme (qui ne dit jamais son nom) bien à soi produit par l'étrange altérité du monde social que l'écrivain met à distance en réinventant la réalité de ses fictions. 